9th International Symposium on High Energy Spin Physics

Bonn, Germany

September 6-15, 1990

BNL- -45013

DE90 017636

\title{
The New Conceptual Design of Snakes and Spin Rotators in RHIC*
}

\author{
S.Y. Lee and E.D. Courant \\ Accelerator Development Department \\ Brookhaven National Laboratory \\ Upton, NY 11973 USA
}

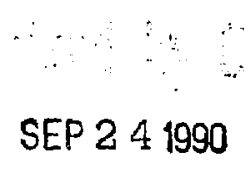

\section{ABSTRACT}

We discuss the generalized snake configurations, which offers either the advantages of shorter total snake length and smaller horizontal orbit displacement in the compact configuration or the dual functions of a snake and a $90^{\circ}$ spin rotation for the helicity state. The generalized snake is then applied to the polarized proton collision in RHIC. The possible schemes of obtaining high luminosity are discussed.

\section{Introduction} as

Recently, K. Steffen ${ }^{1}$ has discovered a family of snakes with the magnet sequence

$$
S=(-H,-V, 2 H, 2 V,-2 H,-V, H)
$$

where $H$ and $V$ are respectively the horizontal and vertical bending magnets. To satisfy the snake criteria, the sequence of magnets must not alter the particle orbit outside the snake and the spinor of the particle is transformed according to $e^{i \frac{\varphi_{2}}{2} \hat{n}_{a} \cdot \vec{\sigma}}$, where $\varphi$ is the spin rotation angle and $\hat{n}_{s}=\left(\cos \varphi_{s}, \sin \varphi_{s}, 0\right)$ is the snake axis. A $100 \%$ snake rotates the spin with angle $\varphi=\pi$. A $90^{\circ}$ spin rotation corresponds to $\varphi=\pi / 2$. A partial snake corresponds to $|\varphi|<\pi$.

An interesting feature of the Steffen's snake is that the snake axis $\varphi$, and the spin rotating angle $\varphi$ can be varied by varying the excitation of $H$ and $V$ magnets, i.e. for $\varphi=\pi$, we obtain

$$
\begin{aligned}
& \cos ^{2} \psi_{y}+\cos 2 \psi_{x} \sin ^{2} \psi_{y}=0 \\
& \sin \varphi_{y}=\sqrt{2} \cos \psi_{x}
\end{aligned}
$$

where $\psi_{x}$ and $\psi_{y}$ are respectively the spin rotation angle of $H$ and $V$ magnets. The relation between $\psi_{x}$ and $\psi_{y}$ in Eq. (2) ensures the snake condition of the magnet sequence and Eq. (3) determines the snake axis. Figure 1 shows $\psi_{x}, \psi_{y}$ relationship of Eq. (2) and $\varphi_{s}$ vs. $\psi_{y}$. When $\varphi_{s}=0$, or $\pi$, the snake axis is along the radial $\hat{x}$ axis. The total integrated magnet strength is given by

$$
\int B d \ell=1.746\left(6 \psi_{x}+4 \psi_{y}\right)[\mathrm{T}-\mathrm{m}]
$$

"Work performed under the auspices of the U.S. Department of Energy 


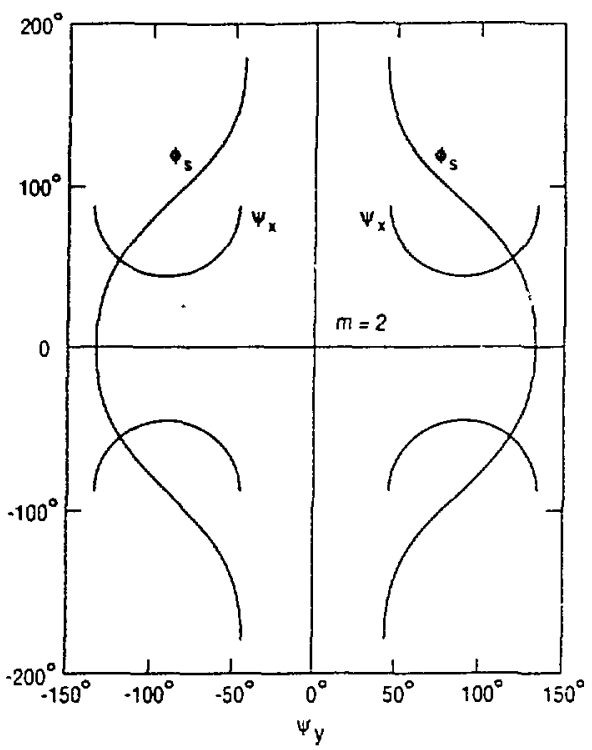

Figure 1: Spin rotation angle of $H, V$ magnets, $\psi_{x}, \psi_{y}$ is shown for $100 \%$ snake. and the corresponding orbit displacements are given by

$$
D_{x}=\left(\ell_{x}+\ell_{y}+2 \ell_{g}\right) \frac{\psi_{x}}{G \gamma} ; D_{y}=\left(2 \ell_{x}+\ell_{y}+2 \ell_{g}\right) \frac{\psi_{y}}{G \gamma}
$$

where $\ell_{x}, \ell_{y}$ and $\ell_{g}$ are respectively the length of $H$ and $V$ magnets and the distance between the adjacent magnets and where $G=(g-2) / 2$ is the Pauli anomalous $g$ factor. Since the lengths of the magnets $\ell_{x}, \ell_{y}$ are given by

$$
\ell_{x, y}=1.746 \frac{\psi_{x, y}}{B[T]}
$$

where the magnetic flux density $B$ is in Tesla, therefore the orbit displacement is inversely proportional to the magnetic field strength of the dipole.

The advantage of the Steffen snake is that the snake axis $\hat{n}_{3}$ can be changed continuously by proper $\psi_{x}, \psi_{y}$ excitations. However, the snake configuration suffers the rigid structure of magnet position, i.e. the total length of the snake is given by

$$
L=6 \ell_{x}+4 \ell_{y}+6 \ell_{g}+\left[\ell_{x}+2 \ell_{g}\right],
$$

where extra free space in the bracket of Eq. (7) is wasted. On the other hand, if the snake can be divided into two parts, then the snake can be fitted into two adjacent straight sections. In this case, the helicity state of the particles at the mid-section of the snake can be achieved. Such a modified snake configuration has been worked out recently. ${ }^{2}$ In this paper, we apply the idea to study the possible polarized proton collision mode in RHIC (the Relativistic Heavy Ion Collider). Section 2 reviews the modified snake configuration. Section 3 discusses the AGS-RHIC requirement. The conclusion is in Section 4.

\section{Review of the Modified Snake Configuration}

The essential feature of the Steffen snake is the symmetric arrangement of vertical bending magnets and the anti-symmetric horizontal bending magnets. These features 
can be preserved in the following modified snake configuration

$$
S_{m}=(-H,-V, m H, 2 V,-m H,-V, H)
$$

where the number $m$ is determined by the geometry. Figure 2 shows the schematic drawing of the magnet arrangement, where $m$ is given by the geometric condition

$$
(m-1)\left(d+\frac{1}{2}(m-1) \ell_{x}+\ell_{y}+\ell_{g}\right)=\ell_{x}+\ell_{y}+2 \ell_{g} \text {. }
$$

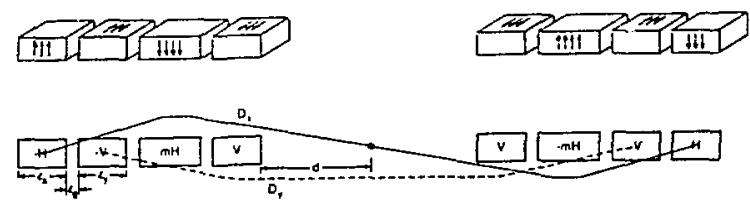

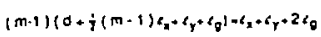

Figure 2: Schematic drawing of the modified snake configuration.

The spin rotation angle $\varphi$ and the snake axis angle $\varphi$, are given by

$$
\begin{aligned}
& \cos \frac{\varphi}{2}=\cos ^{2} \psi_{y}+\cos m \psi_{x} \sin ^{2} \psi_{y} \\
& \cos \varphi_{s}=\frac{-\sin \frac{m \psi_{x}}{2} \cos \psi_{y}}{\sqrt{\cos ^{2} \frac{m \psi_{x}}{2}+\sin ^{2} \frac{m \psi_{x}}{2} \cos ^{2} \psi_{y}}}
\end{aligned}
$$

Note that $m \psi_{x}$ and $\psi_{y}$ are the relevant variable in determining $\varphi$ and $\varphi_{9}$.

\subsection{The Compact Snake Configuration}

The compact snake configuration corresponds to the parameter $d=0$. Depending on $\ell_{x}, \ell_{y}$ and $\ell_{g}, m$ can be determined from the geometric consideration. For example, assuming $B=2$ Tesla, $\ell_{g}=0.15 \mathrm{~m}$ and $\varphi_{s}=180^{\circ}$, we obtain $\mathrm{m}=2.334$ with a total length of 11.48 meters for the compact snake configuration in comparison with a total length of 13.54 meters for $\mathrm{m}=2$ configuration. The compact snake configuration saves about $15 \%$ of the total length. Besides, the total $\int B d \ell$ and $D_{x}$ are also reduced.

\subsection{Split Snake Configuration}

By adjusting the parameter $\mathrm{m}$, we can obtain proper distance $2 d$ between the two halves of the snake. The orbit displacements at the middle of two halves of the snake can be corrected by a orbit shifter of $\left(-V^{\prime}, V^{\prime}\right)$ at both ends of the snakes. However when the distance $2 d$ becomes large, we will have $m \simeq 1$. Obviously, the total length of the snake and the radial orbital displacement increase as well. Such a split snake configuration would not be practical for the insertion detector area. However the split 
snake can be fitted into two adjacent straight sections separated by a quadrupole. Such a snake configuration ease the design criteria of the low energy ( $\leq 30 \mathrm{GeV}$ ) accelerators.

To fit a collider IR onto the space between the split snake, the snake configuration shown in Fig. 3 is most appropriate.

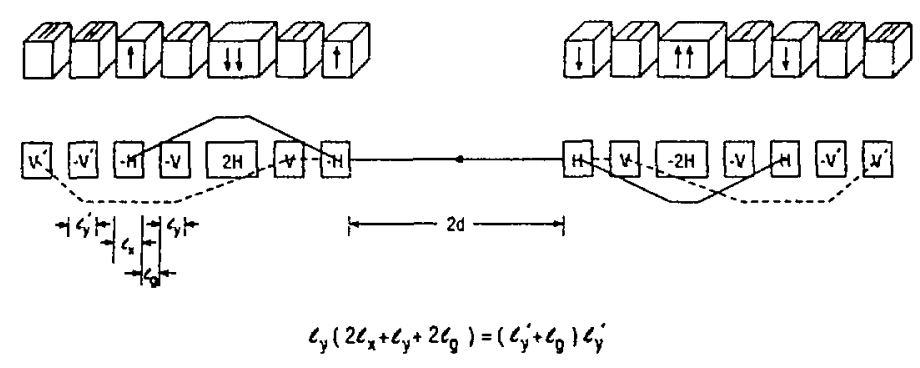

Figure 3: Schematic drawing of the split snake configuration.

The advantage of the split snake configuration is that the spin in the mid section of the snake will be on the horizontal plane. Thus such a snake serves a dual purpose of being a snake and a spin rotator for the helicity state experiments. For a spin up particle passing through the half snake, the spin orientation becomes

$$
S_{x}=-\sin m \psi_{x} \sin \psi_{y} ; S_{y}=\sin ^{2} \frac{m \psi_{x}}{2} \sin 2 \psi_{y} ; S_{x}=0
$$

where $m=2$ for the snake configuration shown in Fig. 3. Because of the horizontal orbit compensation magnet, the spin is further rotated $\psi_{x}$ angle around the vertical axis. Thus by changing $\psi_{x}, \psi_{y}$ excitation, one can study helicity experiments or transverse spin experiments in the same IR. Such a scheme can save the need of four spin rotators in the polarized proton experiment.

\section{Polarized Proton Collision in RHIC}

To achieve a good luminosity in the polarized proton collision in RHIC, we must try to achieve the following parameters:

$$
N_{B} \geq 10^{11} ; \epsilon_{N} \leq 20 \pi \mathrm{mm}-\mathrm{mrad}
$$

where $N_{B}$ is the number of particles per bunch and $\epsilon_{N}$ is the $95 \%$ normalized emittance. To achieve these goals, 20 linac pulses accumulation in the Booster is needed. These polarized protons are then accelerated in AGS to reach $G \gamma \simeq 48$. Partial snake in AGS was studied recently ${ }^{3}$ to correct the imperfection resonances. We foresee no difficulty in reaching $G \gamma=48$ with about $50 \%$ polarization.

Once the polarized proton reach $G \gamma \simeq 48$, the beam can be transferred to RHIC without depolarization. "The depolarization resonance strength in RHIC is shown on Fig. 4, i.e. the important resonance location will be

$$
K=81 n \pm \nu_{B} ; \nu_{B}=\nu_{y}-6
$$

where $\nu_{B}$ is the phase advance accumulated across the dipole cells. The maximum resonance strength will be of the order $\epsilon_{K} \leq 0.5$ for $\epsilon_{N} \leq 10 \pi \mathrm{mm}$-mrad. The imperfection resonance will be of the order 0.06 , when the closed orbit is corrected to within $0.3 \mathrm{~mm}$ 
rms. Careful numerical simulation and analytic study $y^{5}$ indicates that two snakes will be sufficient to maintain the spin polarization in RHIC. Using the concept of Section 2 , one can employ two snakes in each ring. The corresponding luminosity at the top energy of $250 \mathrm{GeV}$ is given by

$$
\mathcal{L}=5.7 \times 10^{31} \frac{\left(\frac{N_{B}}{10^{11}}\right)^{2}\left(\frac{B}{57}\right)^{2}}{\left(\frac{\epsilon_{N}}{20 \pi}\right)^{2}} \mathrm{~cm}^{-2} \mathrm{sec}^{-1}
$$

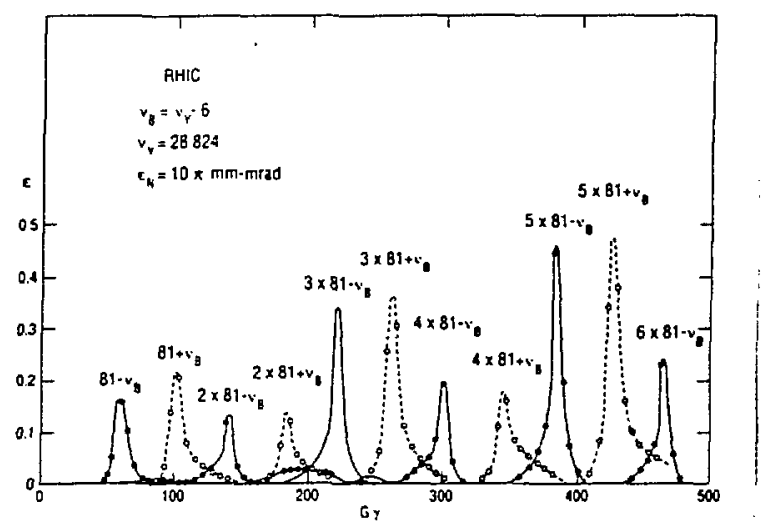

Figure 4: Intrinsic resonance strength for RHIC for $\epsilon_{N}=10 \pi \mathrm{mm}$-mrad.

where $B$ is the number of bunches in each ring. Note here that the importance of smaller emittance in obtaining higher luminosity. The luminosity will gain a factor of 4 when the emittance is smaller by a factor of 2 .

\section{Conclusion}

We have made feasibility study for the possible polarized proton operation in RHIC. The important tasks are:

1. multi-pulses accumulation in the AGS-Booster with small emittance,

2. accelerator through $A G S$ to reach $G \gamma \simeq 48$, where the transfer line between the AGS and RHIC is spin transparent, and

3. construction of four snakes in RHIC.

When these tasks are accomplished, the polarized proton luminosity will be larger than $5 \times 10^{31} \mathrm{~cm}^{-2} \mathrm{sec}^{-1}$ at $\epsilon_{N}=20 \pi \mathrm{mm}-\mathrm{mrad}$. By carefully maintaining the emittance at $10 \pi \mathrm{mm}$-mrad, the luminosity can achieve $2 \times 10^{32} \mathrm{~cm}^{-2} \mathrm{sec}^{-1}$, which corresponds to 2 interactions per crossing.

\section{References}

1. K. Steffen, Particle Accelerator 24, 45 (1989).

2. S.Y. Lee, "Snakes and Spin Rotators", BNL 52248/UC-414 (1990).

3. T. Roser, "Properties of Partially Excited Siberian Snakes", AIP Conf. Proc. No. 187 , p. 1442 (1988).

L. Ratner, ed., Workshop on Partial Snake in AGS. June 1990.

4. S.Y. Lee and E.D. Courant, "Effect of the Vertical Bends in ATR Transfer Line on the Polarized Proton Operation", AD/RHIC-63 (1990).

5. S.Y. Lee and E.D. Courant, Phys. Rev. D41, 292 (1990). 